\title{
Hippocampal-Prefrontal Cortical Circuit Mediates Inhibitory Response Control in the Rat
}

\author{
Yogita Chudasama, Victoria M. Doobay, and Yuchen Liu \\ Department of Psychology, McGill University, Montreal, Quebec H3A 1B1, Canada
}

We investigated the interdependent function of the ventral hippocampus (vHC) and ventral prefrontal cortex (vPFC) in visuospatial attention and inhibitory control using a disconnection lesion approach. Rats were trained, and several aspects of their cognitive performance tested on the 5-choice reaction time task. The animals were prepared with unilateral lesions of both the vPFC and vHC, either in the same hemisphere ("ipsilateral") or in opposite hemispheres ("disconnection"). The disconnection lesion led to both impulsive and compulsive behavior. This deficit is reminiscent of the effects of either bilateral vPFC or bilateral vHC lesions on the same task and is thought to reflect the bihemispheric disruption of the hippocampal-prefrontal circuit. With ipsilateral lesions, behavioral deficits were transient or absent altogether, suggesting that the intact hemisphere was able to exert near normal levels of behavioral control. These behavioral effects were observed in the absence of any changes to visual attention, speed of response, or general motivation. This study provides evidence that optimal inhibitory control of behavior draws upon the functional interaction between the vHC and vPFC.

\section{Introduction}

In rats, the ventral part of the prefrontal cortex includes the ventral prelimbic, infralimbic, and medial orbital cortex (Heidbreder and Groenewegen, 2003). The most pervasive feature of the behavior of animals with ventral prefrontal cortex (vPFC) lesions is disinhibition. Inhibitory control deficits take multiple forms, such as the compulsive repetition of responses or the failure to withhold an impulsive act. Rats and mice with excitotoxic lesions of the vPFC (or subregions thereof) exhibit difficulty in tasks that require the active suppression of inappropriate responses (for review, see Chudasama, 2011; Dalley et al., 2011). Lesions to the hippocampus, a structure that is most often associated with spatial memory, also affect behavioral control. In fact, ablation of the hippocampus, much like that of the vPFC, causes rats to become disinhibited under a range of testing conditions (Jarrard and Isaacson, 1965; Nonneman et al., 1974; Gray and McNaughton, 1983; Rawlins et al., 1985). A potential explanation for the similarity of these effects is that the hippocampus and vPFC work closely together in controlling certain aspects of executive function.

In line with this speculation, neuroanatomical tracing studies demonstrate robust projections from the hippocampus to the prefrontal cortex. In rats, neurons in area CA1/subiculum of the

\footnotetext{
Received March 26, 2012; revised June 11, 2012; accepted June 14, 2012.

Author contributions: Y.C. designed research; Y.C., V.M.D., and Y.L. performed research; Y.C., V.M.D., and Y.L. analyzed data; Y.C. wrote the paper.

This work was supported by grants from the Natural Sciences and Engineering Research Council of Canada and the Canadian Foundation for Innovation. We thank Emma Valence and Debra Langleben for behavioral testing, and Sean Hassan for his help with data analysis. We are very grateful to Andrew R. Abela, Alana Knapman, David A. Leopold, and Norman M. White for helpful comments on the manuscript.

The authors declare no competing financial interests.

Correspondence should be addressed to Dr. Yogita Chudasama, Laboratory of Brain and Behaviour, Department of Psychology, McGill University, Montreal, QC H3A 1B1, Canada. E-mail: yogita.chudasama@mcgill.ca.

DOI:10.1523/JNEUROSCI.1463-12.2012

Copyright $\odot 2012$ the authors $\quad 0270-6474 / 12 / 3210915-10 \$ 15.00 / 0$
}

ventral hippocampus (vHC) project to the medial orbitofrontal, infralimbic, and prelimbic areas of the vPFC. In contrast, projections from the dorsal hippocampus to the vPFC appear significantly weaker (Jay and Witter, 1991; Verwer et al., 1997; Ishikawa and Nakamura, 2006). This difference in connectivity complements the finding that ventral but not dorsal hippocampal lesions produce behavioral disinhibition (Abela et al., 2012). Interestingly, the hippocampal-prefrontal projections are not reciprocal; direct projections from the prefrontal cortex to the hippocampus have not been reported. Nonetheless, there is likely to be indirect transmission of prefrontal signals to the hippocampus through relays in the midline thalamus (Wouterlood et al., 1990; McKenna and Vertes, 2004; Vertes et al., 2006; Prasad et al., 2012). Furthermore, both the $\mathrm{vHC}$ and $\mathrm{vPFC}$ provide direct input to the nucleus accumbens (French and Totterdell, 2002, 2003). Thus, anatomical studies support a range of pathways by which hippocampal and prefrontal circuitry can interact in the control of behavior. The functional viability of these pathways has been supported using electrical microstimulation (for review, see Thierry et al., 2000; Grace et al., 2007). However, despite the evidence to suggest that the $\mathrm{vHC}$ and $\mathrm{vPFC}$ operate together within a rich anatomical network, the functional interaction between these structures has not been experimentally addressed with behavioral testing.

In this study we approach this topic by measuring different forms of response inhibition following combined unilateral excitotoxic lesions of the vHC and vPFC. After "disconnection," where a lesion of vPFC in one hemisphere was combined with that of a vHC lesion in the opposite hemisphere, rats displayed both impulsive and compulsive behavior in the 5-choice reaction time task. In contrast, following "ipsilateral" lesions to both structures in the same hemisphere, animals were much less affected, suggesting that the intact hemisphere was able to exert near normal levels of behavioral control. We discuss these find- 
ings in the broad context of forebrain control over behavioral inhibition.

\section{Materials and Methods}

Subjects

All subjects were male Long-Evans rats (Charles River) housed in pairs in a temperature-controlled room $\left(22^{\circ} \mathrm{C}\right)$, under diurnal conditions $(12 \mathrm{~h}$ light/dark). Rats were food deprived and maintained at $85 \%$ of their free-feeding weights throughout the experiment. All testing occurred at a regular time during the light period and animals were $250-275 \mathrm{~g}$ at the start of behavioral training. The rats were tested as two separate cohorts. The first cohort comprised 28 rats and the second cohort comprised 21 rats. All experimental procedures were approved by the McGill University Animal Care Committee, in accordance with the guidelines of the Canadian Council on Animal Care.

\section{Apparatus}

The test apparatus (Fig. 1) consisted of four $25 \times 25 \mathrm{~cm}$ aluminum chambers (Lafayette Instruments). The rear wall of each chamber was concave and contained nine apertures, each $2.5 \mathrm{~cm}$ square, $4 \mathrm{~cm}$ deep, and set $2 \mathrm{~cm}$ above floor level. Each aperture could be blocked with a metal cover when not required. For the present task, aperture numbers 1 , $3,5,7$, and 9 were open. Nosepoke responses in the apertures were detected by photocells located at the entrance of each aperture. Each aperture was illuminated by a standard $3 \mathrm{~W}$ bulb located at the rear of the aperture. The food magazine was located on the rear wall and was attached to a pellet dispenser. The food magazine could be illuminated with a white light emitting diode. In addition, food magazine entries were detected by photocells.

The four chambers were individually housed within soundattenuating cabinets and were ventilated by fans, which also served to mask extraneous background noise. Each chamber was illuminated by a $3 \mathrm{~W}$ house-light mounted in the center of the roof. Sucrose pellets served as food reward (Dustless Precision Pellets; Bioserve). The apparatus and on-line data collection for each chamber were controlled by a Dell computer connected to an Animal Behavior Environmental Test system (Lafayette Instruments) using the Whisker control system for research (Cardinal and Aitken, 2010).

\section{Behavioral procedure}

Although the rats were tested as two separate cohorts, the order and timing of training and testing were identical for both cohorts to ensure that all rats shared the same experimental history.

Habituation and magazine training. Rats were initially given two sessions $(30 \mathrm{~min})$ in which they were allowed to habituate to the testing chamber and collect sucrose pellets from the food magazine. In the first session, the house light and food magazine light were illuminated and $10-15$ sucrose pellets were freely available in the food magazine. In the second session, the house light and food magazine light were illuminated and pellets were delivered into the central magazine according to a variable interval schedule of $15 \mathrm{~s}$. Once rats were reliably retrieving and consuming pellets, they were ready for behavioral training.

Behavioral training. Rats were trained to detect a brief visual stimulus presented randomly in one of five spatial locations. Figure 1 provides a timeline of a single trial and a summary of the possible response types and their corresponding behavioral measures. At the beginning of each test session, the house light was illuminated and a free single pellet was delivered to the food magazine. The first trial was initiated when the rat collected the sucrose pellet from the food magazine. After a fixed $5 \mathrm{~s}$ intertrial interval (ITI), the light at the rear of one of the apertures was illuminated for a short period ( $0.5 \mathrm{~s})$. Responses in this aperture during illumination and for $4.5 \mathrm{~s}$ afterward (the response window) were rewarded with the delivery of a single pellet and a correct response was recorded. Responses in a nonilluminated hole during the signal period (incorrect response) and failures to respond within the response window (omission) resulted in a period of darkness during which all lights were extinguished for $5 \mathrm{~s}$ (time out). Responses in the apertures during the ITI (i.e., before stimulus presentation) were recorded as premature responses and resulted in a time-out period. Following a correct response, additional responses into the apertures (in lieu of retrieving the reward) were recorded as perseverative responses. A response in the food magazine after the delivery of a sucrose pellet, or after the time-out period, initiated the next trial.

During any one session, the light stimulus was presented an equal number of times in each of the five apertures in a random order. A daily session consisted of 100 trials or was terminated after 30 min of testing. For the first session of training, the stimulus duration and response window were both set at $60 \mathrm{~s}$. These variables were altered on subsequent sessions according to the individual animal's performance until the target set of task parameters could be instituted. The target parameters were as follows: stimulus duration, $0.5 \mathrm{~s}$; ITI, $5 \mathrm{~s}$; response window, $5 \mathrm{~s}$; timeout period, $5 \mathrm{~s}$. The animals were considered to have reached criterion when these target parameters were attained on five consecutive sessions: $>70 \%$ correct responses and $<20 \%$ omissions within the $30 \mathrm{~min}$ session time. Once rats had acquired this training criterion, they were ready for surgery.

Two weeks following surgery, the rats were tested across 10 sessions on the standard baseline schedule of the task. At the completion of the baseline schedule, a variety of task manipulations were instituted to challenge attentional and inhibitory requirements. First the stimulus duration was reduced from 0.5 to $0.2 \mathrm{~s}$ in one session. Second, rats were exposed to a session of variable long ITIs $(5,7,9$, and $11 \mathrm{~s})$. The session length of the variable long ITI challenge was increased to $45 \mathrm{~min}$. Each ITI was presented pseudorandomly 25 times during the 100-trial session. Each manipulation was preceded by at least one session in which the standard parameters were restored to ensure that the rats returned to their baseline performance. Approximately 2 months postsurgery, performance was re-evaluated on the standard baseline schedule of the task for a further $7 \mathrm{~d}$.

Performance measures. Several performance measures were calculated (Fig. 1). (1) Accuracy was measured as the proportion of responses that were correct to the total number of responses (e.g., number of correct responses/total number of responses), expressed as a percentage (chance performance $=20 \%$ ). Thus this measure did not include errors of omission. (2) Errors of omission were considered separately as the percentage of trials in which no response was made. This measure reflects possible failures in detection and also motivational/motor deficits depending on the overall pattern of results. (3) Premature responses were the number of nosepoke responses made in the apertures during the ITI. Such responses were inappropriate, as the rat failed to wait for the stimulus to appear. The frequency of such premature responses is a measure of poor impulse control. (4) Perseverative responses were additional responses in the apertures following a correct trial only. These responses are inappropriate because the rat responds repeatedly, without consequence, instead of collecting its reward. The number of responses in the apertures following an incorrect trial (i.e., during time out) was collected separately for comparison. An increase in responses following correct rewarded trials relative to incorrect unrewarded trials provides a specific measure of compulsive behavior. (5) Response latency was defined as the time between the onset of the light stimulus and the moment at which the animal's nose broke the photocell beam of the hole. (6) Magazine latency was the time between performance of the correct response and the time the rat entered the food magazine to collect its food reward.

\section{Locomotor activity}

Following postoperative testing, locomotor activity was assessed using four standard home cage activity frames. Each home cage was a clear polycarbonate tub $(61 \mathrm{~cm}$ wide $\times 37 \mathrm{~cm}$ long $\times 20.5 \mathrm{~cm}$ high $)$ lined with sawdust, and covered with a barrier filter lid (Ancare). Each home cage was placed within a Cage Rack SmartFrame $(58 \mathrm{~cm}$ wide $\times 60.33 \mathrm{~cm}$ long $\times 2.11 \mathrm{~cm}$ high) equipped with infrared photobeams located on the interior perimeter of the frame. The rat was placed in the activity cage for $2 \mathrm{~h}$. The total number of horizontal beam breaks was recorded using MotorMonitor software, version 5.05 and transmitted to a Dell Optiplex 745 computer (MCS).

\section{Surgical procedures}

All rats were anesthetized with isoflurane gas (4-5\% induction; $1-3 \%$ maintenance) in conjunction with pure oxygen, and placed in a head 

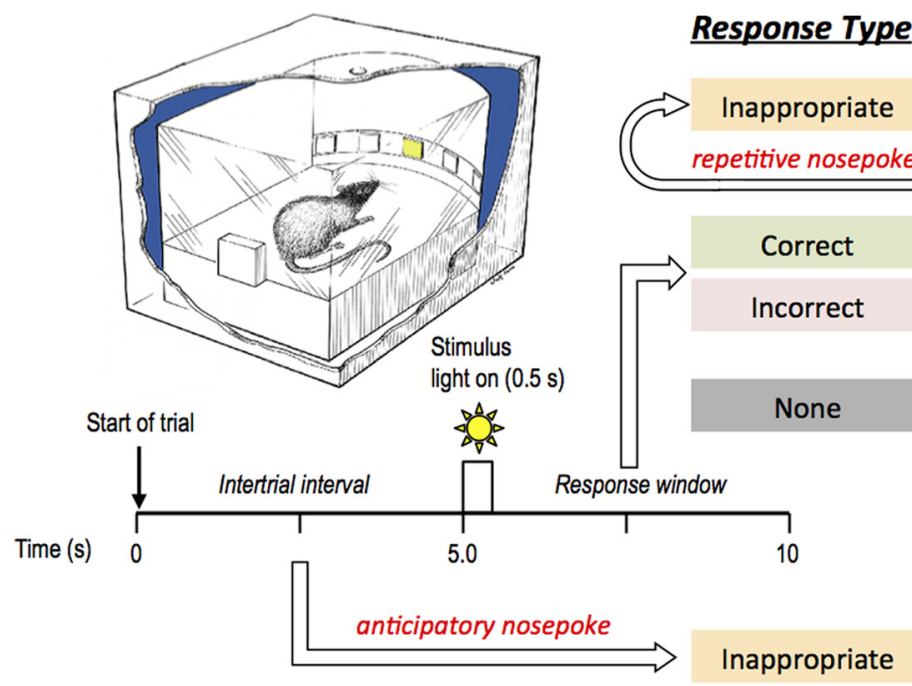

\section{Response Type Behavioral Measure}

Perseverative responses

(compulsivity)

repetitive nosepoke



Correct

Percent accuracy

(performance)

Percent omissions (motor/motivation)

\section{Premature responses (impulsivity)}

Figure 1. Diagram to illustrate the timeline of a single trial in the 5-choice reaction time task, and a summary of the possible response types. Note that inappropriate responses may occur before stimulus presentation (i.e., during ITI) or after stimulus presentation (i.e., during response window) following a correct response. The type of response corresponds to the behavioral measure. Top, left, Provides an illustration of the experimental chamber used for the 5-choice reaction time task in cross section (adapted from original illustration by John Romford, Department of Zoology, Cambridge, UK).

A

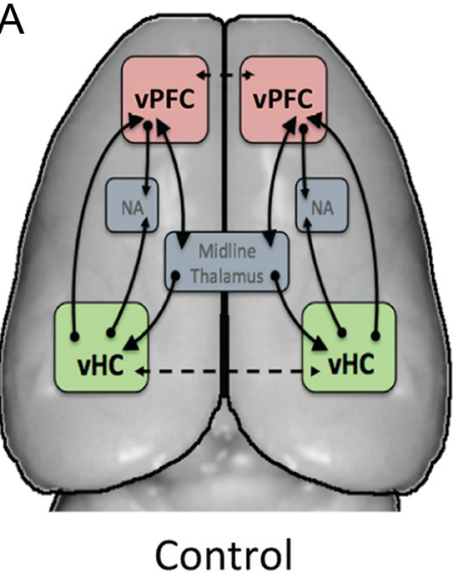

B

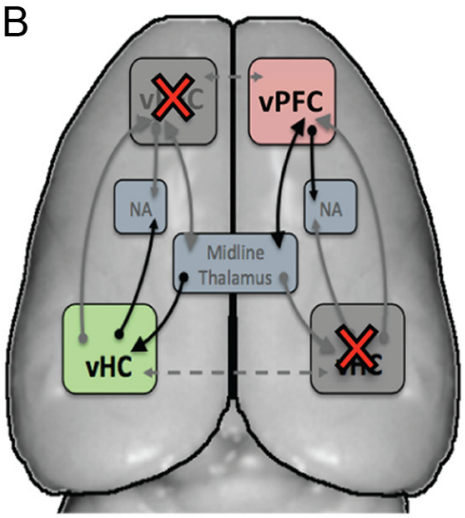

Disconnection Lesions

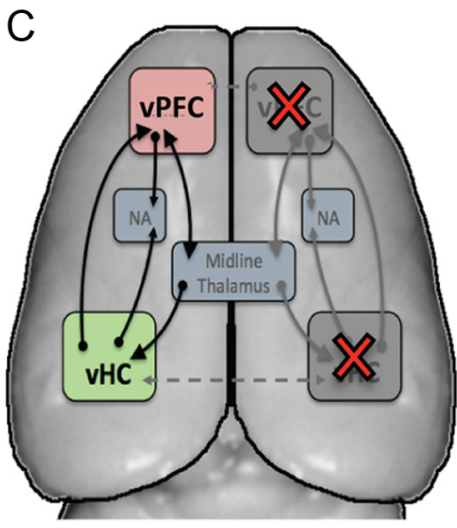

Ipsilateral Lesions

Figure 2. Schematic illustration of experimental design. $\boldsymbol{A}$, Major anatomical connections of the $\mathrm{VPFC}$ and the vHC in the rat brain. $\boldsymbol{B}$, Following combined contralateral vHC and vPFC lesions in opposite hemispheres (i.e., disconnection), a subset of the connections are disrupted in each hemisphere. C, Following combined IPL lesions of the vPFC and vHC, the afferent and efferent projections from the two structures are disrupted in one hemisphere, while the other hemisphere remains intact. Importantly, in the disconnection, all direct and indirect connections between vPFC and vHC are abolished in both hemispheres. Red crosses, lesioned area; solid line, intrahemispheric projection; dashed line, potential interhemispheric projection; NA, nucleus accumbens.

holder fitted with atraumatic earbars in a stereotaxic frame (David Kopf Instruments). The incisor bar was set to $-3.3 \mathrm{~mm}$. The scalp was retracted to expose the skull and craniotomies were made directly above the target region of the brain. A $1 \mu \mathrm{l}$ SGE microsyringe (Canadian Life Science) was used to administer bilateral injections of 0.09 M NMDA (Sigma-Aldrich) dissolved in sterile PBS ( $\mathrm{pH}$ 7.0-7.2). Injection coordinates were taken from the atlas of Paxinos and Watson (2005). Dorsoventral (DV) readings were in reference to the dural surface. Rats that served as sham controls received the same surgical treatment but received injections of saline.

For lesions of the vPFC, rats received two injections of $0.4 \mu \mathrm{l}$ of $0.09 \mathrm{M}$ NMDA at the following anteroposterior (AP), mediolateral (ML), and DV coordinates from bregma: AP: $+4.2 \mathrm{~mm}$; ML: $\pm 0.7 \mathrm{~mm}$; DV: -3.0 $\mathrm{mm}$, and AP: $+2.7 \mathrm{~mm}$; ML: $\pm 0.7 \mathrm{~mm}$; DV: $-3.4 \mathrm{~mm}$. Each injection was made over $2 \mathrm{~min}$, and the injector remained in place for an additional $2 \mathrm{~min}$ for dispersion before it was retracted.

For lesions of vHC, rats received three injections of $0.09 \mathrm{M}$ NMDA at the following coordinates: AP: $-4.6 \mathrm{~mm}$; $\mathrm{ML} \pm 5.0 \mathrm{~mm}$; DV $-6.7 \mathrm{~mm}(0.5 \mu \mathrm{l})$; AP: $-4.7 \mathrm{~mm} ; \mathrm{ML} \pm 4.4 \mathrm{~mm}$; DV $-6.7 \mathrm{~mm}(0.5 \mu \mathrm{l})$; and AP: $-4.8 \mathrm{~mm}$; ML $\pm 4.6 \mathrm{~mm} ; \mathrm{DV}-7.5 \mathrm{~mm}(0.4 \mu \mathrm{l})$. Each injection was made over $2 \mathrm{~min}$, and the injector remained in place for an additional $2 \mathrm{~min}$ for dispersion.

The combined surgical manipulations are schematized in Figure 2. The rats were tested as two separate cohorts. The first cohort comprised a total of 28 rats. Nineteen rats received a disconnection lesion in which a unilateral $\mathrm{vPFC}$ lesion was combined with a contralateral $\mathrm{vHC}$ lesion (the DISC group), and nine rats served as sham controls for this cohort (Fig. 2A,B). The second cohort comprised a total of 21 rats in which 13 rats received an ipsilateral lesion where a lesion to both structures was made in the same hemisphere (the IPL group), thereby leaving one hemisphere intact (Fig. 2C). An additional eight rats served as sham controls. The side on which the lesion was made (right or left hemisphere) was counterbalanced.

Following surgery, animals were administered an injection of carprofen (analgesic) for up to $3 \mathrm{~d}(5 \mathrm{mg} / \mathrm{kg}$, s.c.). To reduce transient seizure activity during postoperative recovery, rats were treated with midazolam $(0.5 \mathrm{mg} / \mathrm{kg}$, i.p.) $5 \mathrm{~min}$ before the end of surgery, and monitored in a recovery cage that was void of extraneous sensory stimulation (e.g., excessive bright lights and loud noise). 
A

Ipsilateral Lesions

Left Hemisphere
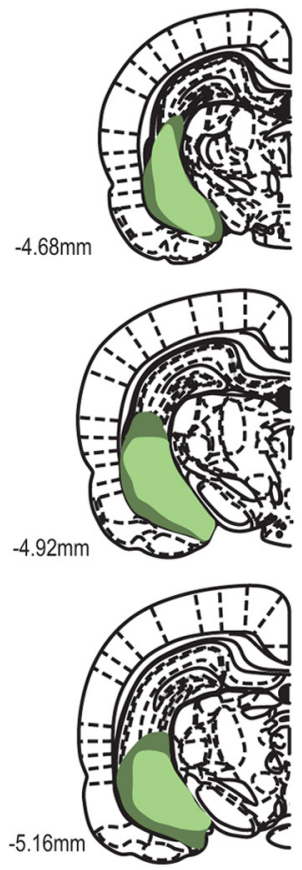
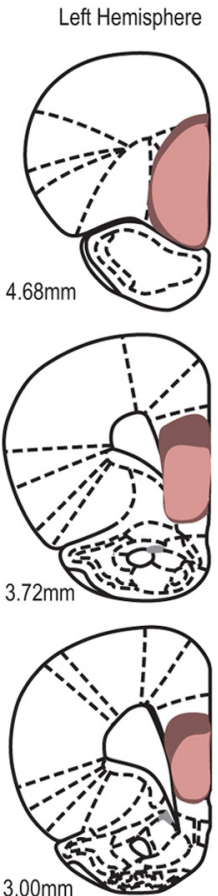

B

Disconnection Lesions

Left Hemisphere


Right Hemisphere
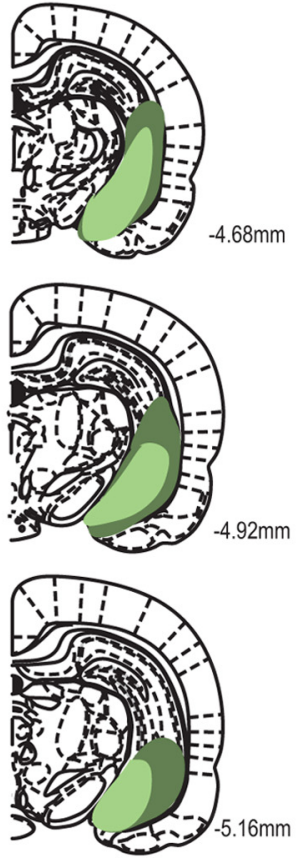

C

Disconnection Lesions

Left Hemisphere Right Hemisphere



$3.72 \mathrm{~mm}$

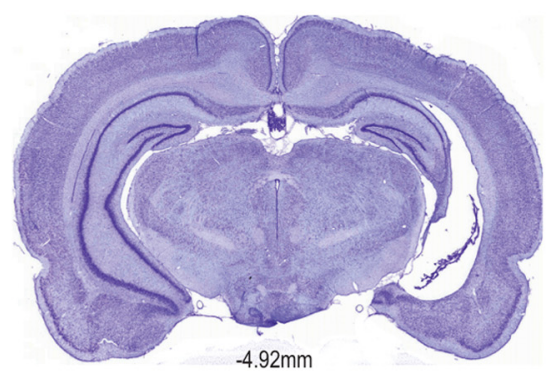

Figure 3. Diagrammatic reconstruction of coronal sections of rat brain showing the largest (dark green) and smallest (light green) extent of the vHC lesion, and the largest (dark red) and smallest (light red) extent of the vPFC lesion. $\boldsymbol{A}, \mathrm{vHC}$ (left hemisphere) and vPFC (left hemisphere) lesions in the ipsilateral lesioned group. $\boldsymbol{B}, \mathrm{vPFC}$ (left hemisphere) and vHC (right hemisphere) lesions in the disconnected lesioned group. Side of lesion was counterbalanced for both groups. C, Representative photomicrograph of coronal sections showing extent of neuronal loss in the vPFC (left hemisphere) and vHC (right hemisphere) in a representative rat with a disconnection lesion. Numbers indicate approximate anteroposterior coordinates relative to bregma according to the atlas of Paxinos and Watson (2005).

\section{Histology}

At the conclusion of behavioral testing, the rats were perfused transcardially with $0.9 \%$ saline followed by $4 \%$ formal saline. After dehydration by immersion in $20 \%$ sucrose, the brains were sectioned on a cryostat at $40 \mu \mathrm{m}$ thickness. Every other section was mounted on glass slides and stained with cresyl violet. The sections were used to verify the location of the lesion and to assess the extent of lesion-induced neuronal loss.

\section{Data analysis}

Data for each variable were subjected to a repeated-measures ANOVA using the PASW statistical package, version 18.0 (PASW). Data were initially explored using "boxplots" and tests of homogeneity of variance so that outliers were identified and skewed data, which violate the distribution requirement of the ANOVA, were transformed appropriately (arcsine, square root, or logarithmic) as recommended by Winer (1971). Homogeneity of variance across groups was assessed with the Mauchly sphericity test. When datasets significantly violated this requirement for a repeated measures design, the $F$ term was tested against degrees of freedom corrected by Huynh-Feldt to provide a more conservative $p$ value for each $F$ ratio. Where $F$ ratios were significant, post hoc comparisons were made using Fisher's least significant difference (LSD). The criterion for statistical significance was a probability level of $p<0.05$. The between-subjects factor was lesion. The within-subjects factor was number of sessions ( $10 \mathrm{~d}$ or $7 \mathrm{~d}$ ), stimulus duration at two levels ( 0.5 and $0.2 \mathrm{~s})$, and ITI at four levels $(5.0,7.0,9.0$, and $11.0 \mathrm{~s})$. The within-subjects factor for locomotor activity was the number of blocks of $15 \mathrm{~min}$ over $2 \mathrm{~h}$ (i.e., eight blocks).

\section{Results}

The combined $\mathrm{vHC}$ and $\mathrm{vPFC}$ lesions were performed after the rats had been trained to criterion performance in the 5-choice reaction time task. Two weeks following postoperative recovery, the two lesions groups and the sham control animals were retested in the same task. The tests were first performed 2 weeks after surgery and then followed up with further testing 2 months after the surgery. In addition to testing animals on the standard baseline schedule, in separate sessions, we examined the effect of manipulating the duration of the stimulus, and the timing of stimulus presentation on the behavior of the various groups. Each of these aspects of the study is described in turn in the following sections.

\section{Combined lesion approach and histological verification}

The DISC and IPL combined lesions and the sham control surgery are schematized in Figure 2. Briefly, in rats with DISC lesions, ablation of $\mathrm{vPFC}$ and $\mathrm{vHC}$ in opposite hemispheres abolishes all intrahemispheric functional interaction between the two structures (Fig. 2B). In contrast, the IPL lesions, which also involves unilateral ablation of the same structures, leaves one hemisphere entirely intact (Fig. 2C). Therefore, any observed differences between these two conditions can be interpreted as reflecting the intrahemispheric interaction between the two structures (see Discussion).

The extent of the lesions across all groups are summarized in Figure 3, $A$ and $B$, with cytoarchitectonic borders and nomenclature adopted from Paxinos and Watson (2005). Figure 3C provides a representative photomicrograph of a DISC lesion where the $\mathrm{vPFC}$ and $\mathrm{vHC}$ lesions were made in opposite hemispheres. Animals with lesions made to the vPFC showed extensive cell damage, which began at the frontal pole and included the rostromedial extent of the orbitofrontal cortex. The lesion began at bregma $+5.64 \mathrm{~mm}$ and extended caudally to bregma $+2.52 \mathrm{~mm}$. At its most rostral level, the lesion included the prelimbic cortex $(4.68-4.20 \mathrm{~mm}$ from bregma). From bregma $3.72 \mathrm{~mm}$, the lesion was centered on the ventral portion of the prelimbic cortex and 
the infralimbic cortex. Cell loss was also observed in the dorsal peduncular cortex, located immediately ventral to the infralimbic cortex. In one case, the lesion encroached into the rostral extent of the dorsal pregenual anterior cingulate cortex (Cg1) but was spared as the lesion extended caudally. The animal was accepted into the behavioral analysis if the vPFC lesion showed almost complete destruction of the pyramidal cells of the rostromedial orbital, ventral prelimbic, and the infralimbic cortex of the vPFC. Rats with vPFC lesions that were too small or incomplete or were extensive and included the rostral and caudal dorsal anterior cingulate cortex ( $\mathrm{Cg} 1$ and $\mathrm{Cg} 2)$ were removed from statistical analysis.

Rats with vHC lesions showed extensive cell loss in the ventral portion of the hippocampus. The lesion extended rostrocaudally from $\mathrm{AP}-4.36 \mathrm{~mm}$ to $-5.40 \mathrm{~mm}$ from bregma, and included the pyramidal cells of the cornu ammonis (CA) fields (CA1, CA2, and CA3), the dentate gyrus (molecular and granular layers), and the ventral subiculum. In most rats, the vHC lesion encroached the ventral portion of the dorsal hippocampus. In all cases, the amygdala and structures lateral to the $\mathrm{vHC}$ such as the parahippocampal region including entorhinal and perirhinal cortex were spared. Rats that showed sparing of the $\mathrm{vHC}$, or minimal damage restricted to the inner pyramidal layer of the $\mathrm{vHC}$, were excluded from statistical analysis.

In cohort one, of the 19 rats that received disconnected contralateral lesions of the $\mathrm{vPFC}$ and $\mathrm{vHC}, 3$ rats were excluded because the lesion (vPFC, vHC, or both) was too small or extensive in size. An additional three rats did not recover from surgery. One rat from the sham group was removed from analysis because he failed to complete a sufficient number of trials during task performance (e.g., 2 of 100 trials). Thus, the final numbers for the contralateral disconnection analysis were sham, 8 and DISC, 13.

In cohort two, of the 13 rats that received ipsilateral lesions of the vPFC and vHC in the same hemisphere, 7 rats were discarded from analysis: 3 rats presented with a small or incomplete lesion to either the $\mathrm{vPFC}$ or $\mathrm{vHC}, 1$ rat showed extensive damage to the basolateral amygdala, and 3 rats did not recover from surgery. The final group numbers for the IPL lesion analysis were sham, 8 and IPL, 6.

\section{Postoperative behavior: two weeks postlesion}

Preliminary analysis of the sham data revealed that the sham rats in each cohort (sham-DISC and sham-IPL) did not differ in performance across the 10 postoperative baseline sessions (accuracy: $F_{(1,14)}=1.13 ; p>0.05$; premature response: $F_{(1,14)}=0.42 ; p>$ 0.05 ; perseverative response: $F_{(1,14)}=0.53 ; p>0.05$; response latency: $F_{(1,14)}=3.02 ; p>0.05$; magazine latency: $F_{(1,14)}=0.20$; $p>0.05)$. Although there was a trend for the number of omissions to be higher in the sham-DISC group $\left(F_{(1,14)}=4.29 ; p=\right.$ $0.06)$, there was a decline in the number of omissions with increasing number of sessions $\left[F_{(9,126)}=5.86, p<0.0001\right.$; mean $( \pm \mathrm{SEM})$ percentage omissions on session 1: sham-IPL 18.7 ( \pm 6.8 ), sham-DISC 24.5 ( \pm 5.2 ); mean percentage omissions on session 10: sham-IPL, $7.3( \pm 3.8)$; sham-DISC, $10.3( \pm 2.8)]$. Therefore, all of these animals were treated as a single sham group ( $n=16)$ for subsequent analyses.

The animals were tested over 10 sessions, with several behavioral measures plotted for each session in Figure $4 A-C$. The overall response accuracy was unaffected by either lesion, as can be seen in Figure $4 A\left(F_{(2,32)}=0.90 ; p>0.05\right)$. Nor did the lesions affect the rate of omission $\left(F_{(2,32)}=0.75 ; p>0.05\right)$ or response latency $\left(F_{(2,32)}=1.12 ; p>0.05\right)$. The lesions did, however, affect the rate of premature responding across groups (Fig. 4B)
$\left(F_{(2,32)}=3.21 ; p<0.05\right)$. Both the DISC and IPL groups showed an elevation of premature responses 2 weeks postlesion, but only the DISC group responses were significantly different from the sham controls (LSD $p=0.02$ ), whereas the IPL group failed to reach significance (LSD $p=0.09$ ). Further comparisons revealed that the DISC group was not significantly different from the IPL group (LSD $p=0.96)$. A much larger effect was found in the rate of perseverative responses, which was substantially elevated in the DISC group (Fig. 4C). Perseverative responses were additional responses in the apertures following a "correct" trial. These responses were inappropriate because the rat responded repeatedly, without consequence, instead of collecting its reward. A repeated-measures ANOVA on the number of perseverative responses revealed a significant main effect of lesion [mean $( \pm \mathrm{SEM})$ responses following correct trials: sham $15.9( \pm 2.5)$, IPL 11.9 ( \pm 4.4$)$, DISC 55.6 ( \pm 12.5$) ; F_{(2,32)}=10.15 ; p<0.0001$ ], session $\left(F_{(4,119)}=4.93 ; p<0.001\right)$, and lesion $\times$ session interaction $\left(F_{(7,119)}=2.07 ; p<0.05\right)$. Post hoc comparisons confirmed that rats with DISC lesions were highly perseverative relative to rats in the sham group (LSD $p=0.001$ ) and the IPL group (LSD $p=0.002$ ). Importantly, rats with DISC lesions did not make additional responses in the apertures following an incorrect trial [mean $( \pm$ SEM) responses following incorrect trials: sham $8.2( \pm 2.0)$, IPL $10.8( \pm 4.7)$, DISC $13.5( \pm 4.8) ; F_{(2,32)}=$ $0.74, p>0.05]$. This additional analysis confirms that the DISC lesion produced a specific type of perseveration in which actions, inappropriate to the situation, persisted following a correct rewarded response. Magazine latencies were slightly elevated in the DISC group of rats [mean ( \pm SEM) latency (in seconds) for 10 postoperative baseline sessions: sham, 1.51 ( \pm 0.14 ); IPL, 1.62 $( \pm 0.26)$; DISC, $2.46( \pm 0.85)]$ but the groups did not differ significantly on this measure $\left(F_{(2,32)}=0.91 ; p>0.05\right)$.

\section{Effect of reducing the stimulus duration}

Reducing the stimulus duration to $0.2 \mathrm{~s}$ resulted in significant reduction in response accuracy for all rats regardless of lesion group $\left(F_{(1,32)}=35.39 ; p<0.0001\right.$; Fig. $\left.5 A\right)$ but the groups did not differ significantly from each other $\left(F_{(2,32)}=0.10 ; p>0.05\right)$. The number of premature responses increased for all animals when the stimulus duration was reduced $\left(F_{(1,32)}=4.43 ; p<\right.$ $0.05)$. Consistent with their performance during postoperative baseline, the DISC-lesioned rats maintained high levels of premature responding at the 0.5 and $0.2 \mathrm{~s}$ stimulus duration (Fig. $5 B$ ). However, this overall increase in premature responding failed to reach significance in terms of lesion $\left(F_{(2,32)}=2.86 ; p=0.07\right)$. The elevated perseverative responding exhibited by the DISC group was maintained for the shorter stimulus duration (Fig. 5C) $\left(F_{(2,32)}=16.58 ; p<0.0001\right)$, with the DISC group different from the IPL (LSD, $p=0.0001$ ) and sham control (LSD, $p=0.0001$ ) groups. Nonrewarded incorrect trials did not lead to perseveration in any group $\left(F_{(2,32)}=0.31, p>0.05\right)$. No other main effect or interaction was observed for this measure. Importantly, this selective impairment was observed in the absence of any group differences in terms of omissions $\left(F_{(2,32)}=0.16\right.$; $\left.p>0.05\right)$, response latency $\left(F_{(2,32)}=0.09 ; p>0.05\right)$, or magazine latency $\left(F_{(2,32)}=0.67 ; p>0.05\right)$.

\section{Effect of long and variable ITIs}

In this manipulation, rats were exposed to long ITIs that were presented randomly and unpredictably across one test session. Response accuracy was unaffected by either lesion $\left(F_{(2,32)}=0.55\right.$; $p>0.05)$ as shown in Figure $6 \mathrm{~A}$. However, this manipulation increased the rate of premature responding across all groups 
$\left(F_{(3,96)}=167.43 ; p<0.0001\right.$; Fig. $\left.6 B\right)$. There was also a main effect of lesion $\left(F_{(2,32)}=7.06 ; p<0.01\right)$. This difference was attributable to both lesioned groups making more premature responses compared with shams (LSD: DISC vs sham, $p=0.003$; IPL vs sham, $p=0.007$ ). The DISC and IPL group of rats did not differ from each other (LSD, $p=0.75$ ), and there was no group $\times$ ITI interaction $\left(F_{(6,96)}=1.83\right.$; $\left.p>0.05\right)$. However, individual ANOVAs conducted for each ITI confirmed that the DISC and IPL groups made more premature responses at the two longest ITIs (9.0 s ITI: $F_{(2,32)}=9.05$; $p<0.01 ; 11.0$ s ITI: $F_{(2,32)}=6.97 ; p<$ $0.01)$, and a trend toward premature responses at the $7.0 \mathrm{~s}$ ITI $\left(F_{(2,32)}=2.95\right.$; $p=0.06)$.

There was also a main effect of lesion on the number of perseverative responses $\left(F_{(2,32)}=3.45 ; p<0.05\right)$. Although the number of perseverative responses declined with increasing ITI $\left(F_{(1,45)}=5.44\right.$; $p<0.02$ ), Figure $6 C$ shows that overall, rats with DISC lesions maintained a high level of perseverative responding relative to the IPL (LSD, $p=0.03$ ) and sham groups (LSD, $p=0.03$ ). We also observed a significant lesion $\times$ ITI interaction on this measure $\left(F_{(3,44)}=2.86 ; p=0.050\right)$, which was due to high perseveration exhibited by rats with DISC lesions relative to the IPL and sham groups, especially at the $5.0 \mathrm{~s}$ ITI s $\left(F_{(2,32)}=4.12 ; p<0.05\right)$. Perseverative responses at the other ITIs failed to reach significance (7.0 s: $F_{(2,32)}=$ $1.77 ; p>0.05 ; 9.0 \mathrm{~s}: F_{(2,32)}=2.92 ; p=$ $\left.0.06 ; 11.0 \mathrm{s:} F_{(2,32)}=1.53 ; p>0.05\right)$. The number of additional responses in the apertures following incorrect unrewarded trials was equivalent between groups $\left(F_{(2,32)}=2.51, p>0.05\right)$ con-

firming that rats with DISC lesions were only perseverative following correct trials. Other aspects of performance did not change as a function of lesion (i.e., omissions: $F_{(2,32)}=0.86$; $p>0.05$; response latency: $F_{(2,32)}=0.99 ; p>0.05$; magazine latency $\left.F_{(2,32)}=0.21 ; p>0.05\right)$.

\section{Postoperative behavior: two months postsurgery}

Approximately 2 months postsurgery, the rats were retested for $7 \mathrm{~d}$ on the standard baseline schedule of the task to examine the stability of the observed deficits. Figure $4 D$ shows that the lesion did not affect response accuracy 2 months following the lesion $\left(F_{(2,32)}=1.91 ; p>0.05\right)$. Nor did the lesion affect the number of omissions $\left(F_{(2,32)}=0.28 ; p>0.05\right)$, latency to respond $\left(F_{(2,32)}=1.21 ; p>0.05\right)$, and latency to collect food $\left(F_{(2,32)}=\right.$ $0.44 ; p>0.05)$. As expected, the number of premature responses declined with increasing number of sessions $\left(F_{(6,188)}=2.76 ; p<\right.$ $0.05)$, but rats with DISC lesions maintained a significant increase in premature responses $\left(F_{(2,32)}=6.93 ; p<0.01\right)$ as they did 2 weeks following surgery (Fig. $4 E$ ). Post hoc comparisons confirmed that the DISC group of rats made more premature


Figure 4. Mean performance ( \pm SEM) on postoperative baseline performance 2 weeks after surgery $(\boldsymbol{A}-\boldsymbol{C})$ and 2 months after

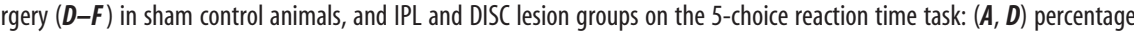
accuracy, $(\boldsymbol{B}, \boldsymbol{E})$ premature responses, and $(\boldsymbol{C}, \boldsymbol{F})$ perseverative responses. ( ${ }^{*} \mathrm{DISC}$ group significantly different from sham and IPL group; ${ }^{\dagger}$ DISC group significantly different from shams, but not IPL group).

responses relative to the other two groups (LSD: DISC vs sham, $p=0.001$; DISC vs IPL, $p=0.026)$. In addition, the increased number of premature responses exhibited by the IPL group early in the course of testing (i.e., two weeks postsurgery) returned to control levels (compare Fig. $4 B, E$ ). Consistent with their disinhibitory nature, Figure $4 F$ shows that rats with DISC lesions maintained a high rate of perseverative responding $\left(F_{(2,32)}=\right.$ $6.12 ; p<0.01)$ across all sessions relative to rats with IPL lesions (LSD, $p=0.01$ ) and sham controls (LSD, $p=0.001$ ). As in previous manipulations, this perseverative effect was specific to correctly rewarded trials only [means ( \pm SEM): sham 23.7 ( \pm 6.3 ), IPL 18.4 ( \pm 6.4$)$, DISC 57.5 ( \pm 13.3$)$ ]; the number of responses in the apertures following an incorrect trial did not differ between groups [means $( \pm$ SEM): sham $7.9( \pm 2.9)$, IPL $12.0( \pm 5.6)$, DISC $\left.8.6( \pm 2.1) ; F_{(2,32)}=0.76, p>0.05\right]$. No other significant effects were observed.

\section{Locomotor activity}

General locomotor activity for each animal was recorded over eight consecutive 15 min blocks. The activity of both groups of rats declined with 



Figure 5. Mean performance ( + SEM) of sham controls, DISC and IPL lesion groups on the 5 -choice reaction time task when the stimulus duration was reduced from 0.5 to $0.2 \mathrm{~s}:(\boldsymbol{A})$ percentage accuracy, $(\boldsymbol{B})$ premature responses, and $(\boldsymbol{C})$ perseverative responses. (*DISC group significantly different from sham and IPL group).

an increasing number of blocks $\left(F_{(6,191)}=75.57 ; p<0.0001\right)$, but there was no main effect of lesion on this measure $\left(F_{(2,32)}=0.03 ; p>0.05\right)$.

\section{Discussion}

This study is the first to demonstrate that mechanisms of inhibitory control involve the functional interaction of an intact $\mathrm{vHC}$ and vPFC. Disconnection of the $\mathrm{vHC}$ and $\mathrm{vPFC}$ in rats resulted in a persistent deficit characterized by increased levels of premature and perseverative responding in the 5-choice reaction time task. In contrast, rats with IPL lesions, in which combined unilateral lesions of the $\mathrm{vHC}$ and $\mathrm{vPFC}$ were restricted to the same hemisphere, showed a transient increase in premature responding. These behavioral effects were observed in the absence of any
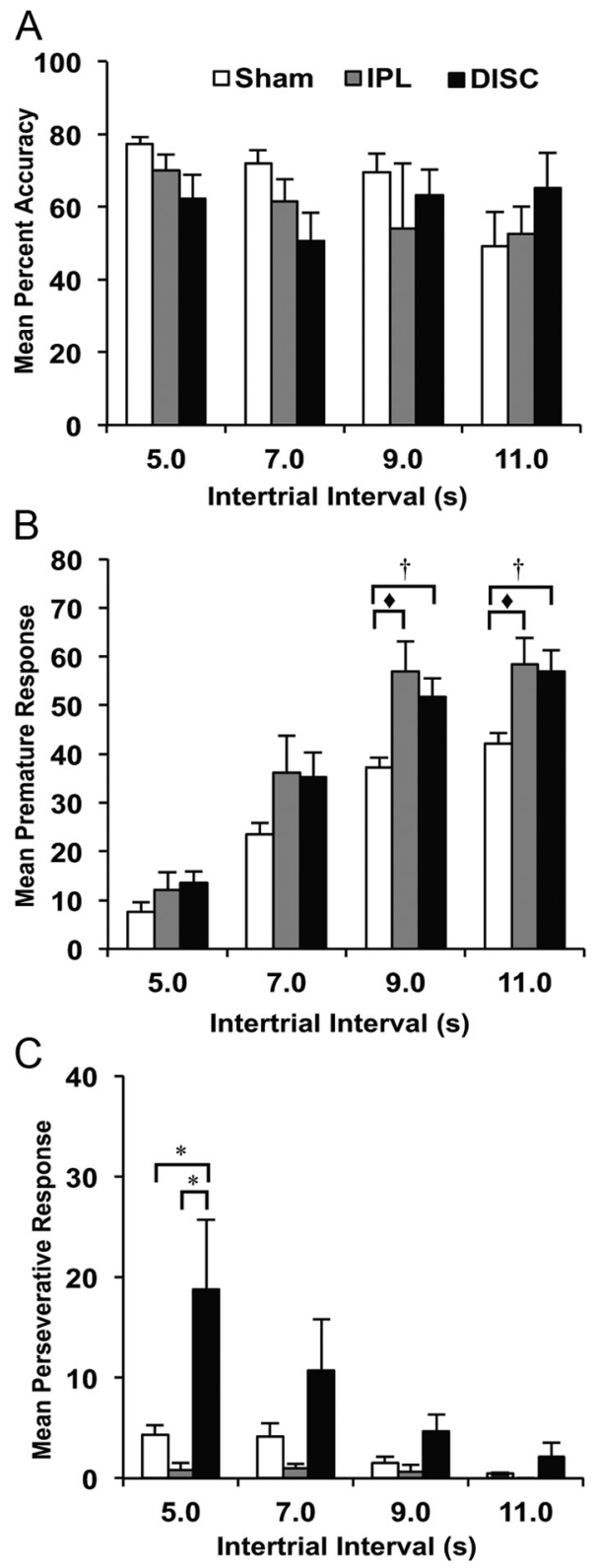

Figure 6. Mean performance ( + SEM) of sham controls, DISC and IPL lesion groups on the 5-choice reaction time task when ITI was made long and variable: $(\boldsymbol{A})$ percentage accuracy, $(\boldsymbol{B})$ premature responses, and $(\boldsymbol{C})$ perseverative responses. ( ${ }^{*}$ DISC group significantly different from sham and IPL group; ${ }^{\dagger}$ DISC group significantly different from sham, but not IPL group; $\diamond$ IPL group significantly different from sham only).

changes to response accuracy (taken as a measure of visual attention), speed of response, or general motivation.

\section{Disconnection as a means to evaluate functional interaction} In animal neuropsychology, a disconnection typically involves a lesion to different brain areas in each hemisphere combined with the resection of forebrain commissures. The latter manipulation assures that the hemispheres operate independently so that a lesion to area $\mathrm{A}$ in one hemisphere and a lesion to area $\mathrm{B}$ in the other will disrupt any process that depends on the functional interaction between A and B. Conversely, if function is preserved under these conditions, it cannot depend on the interaction between these two areas. In our case, there was no transection of the forebrain commissures because in rodents, although it is possible 
(Dunnett et al., 2005), this surgical procedure can be difficult to achieve, and inevitably leads to unwanted collateral damage. Thus, had we not observed a deficit in our DISC group, it would have been impossible to rule out, based on these experiments alone, that interhemispheric connections between the hippocampus and prefrontal cortex were the basis of intact performance. That we observed a deficit in the DISC group which was similar in character to the effects of both bilateral $\mathrm{vHC}$ and bilateral vPFC lesions (Chudasama and Muir, 2001; Passetti et al., 2002; Chudasama et al., 2003; Abela et al., 2012), and was considerably weaker or absent in the IPL group, argues that interhemispheric connections, if present, are insufficient to support normal behavior. Thus on the basis of the DISC manipulation, combined with the appropriate controls, we conclude that behavioral control of inhibition involves the obligatory functional interaction of $\mathrm{vHC}$ and vPFC circuits.

\section{Behavioral effects of $\mathrm{vHC}$ and vPFC disconnection}

There were two primary behavioral effects caused by the DISC lesion. First there was an increase in rats' premature responses during the period in which they anticipated the occurrence of the visual stimulus. Second, there was an abnormal tendency toward perseveration following a correct response, where rats would continue to nosepoke in the apertures following a correct response rather than retrieve their food reward. In addition, the rats maintained their perseverative tendency during the long ITI manipulation even though the DISC lesion led to a decrease in perseverative responding with increasing ITIs, a response pattern that is relatively normal in shams and other lesion groups (e.g., Chudasama et al., 2003). The premature and perseverative forms of response disinhibition were long lasting in that they persisted throughout the course of the experiment and remained present 2 months following the lesion. These effects cannot be attributed to hyperactivity because speed of response and general locomotor activity was in the normal range. Nor can either be ascribed to a general motivational deficit, since they were unaccompanied by omitted trials or a delayed latency to collect food reward. Furthermore, factors such as attention and perception were intact, as evidenced by high response accuracy, even for short-stimulus durations. The data suggest, therefore, that disconnection of the vHC from the $\mathrm{vPFC}$ was specific to response inhibition, leading to a failure to inhibit both premature and perseverative responses.

In contrast to the DISC group, rats with IPL lesions to the vPFC and vHC in the same hemisphere exhibited a transient impulsive deficit, which recovered over time. This deficit may be the result of one of the two unilateral lesions, or indeed a "massaction" effect of the dual lesion. As such, the eventual return to normal impulse control following the ipsilateral lesion may have been mediated by intrahemispheric interactions in the intact hemisphere, whereas the persistent impulsive deficit reflected the lack of intact circuits in either hemisphere. Note that unlike the impulsive effects, the compulsive effects were never observed in the IPL group, arguing against generalized disinhibition due to the combined lesion. Unlike the DISC group, the ipsilateral group did not show an increase in perseverative errors compared with sham controls. This dissociation underscores the distinct forms of response inhibition associated with impulsive behavior (e.g., anticipatory, premature) versus compulsive behavior (e.g., perseveration), and suggests that intact frontohippocampal circuits are particularly relevant to the latter.

However, the IPL group of rats showed a significant impulsive deficit when they were unable to predict the stimulus. Under this condition, the impulsivity exhibited by the IPL group was comparable to that of the DISC group. This finding might imply that a single hemisphere containing intact $\mathrm{vHC}-\mathrm{vPFC}$ circuitry is sufficient to control the inhibition of behavioral impulses, but only when the stimulus can be predicted. It follows that when stimuli are unpredictable, the two hemispheres must work together to exert optimal behavioral control. Surprisingly, the observed impulsivity did not interfere with response accuracy as it does in rats with bilateral vHC lesions (Abela et al., 2012). Evidently, an intact hemisphere can compensate for some aspects of behavior such as control of attention but not the inhibitory control of an impulsive response.

\section{Possible neural basis of inhibitory control deficits}

One interpretation of our data is that the hippocampalprefrontal pathway is a route by which the vHC can influence activity of the vPFC. The qualitatively similar disinhibition of impulse control following bilateral lesions of either area is consistent with this hypothesis (Chudasama et al., 2003; Abela et al., 2012; see also Murphy et al., 2005). Likewise, the elevated perseveration in the present experiments resembles that following bilateral vPFC lesion (Chudasama and Muir, 2001; Passetti et al., 2002; Chudasama et al., 2003), and to some extent bilateral vHC lesions (Abela et al., 2012). Thus direct input from the hippocampal formation through anatomical projections originating in ventral CA1/subiculum might be pivotal in shaping ventral prefrontal function related to behavioral control.

At the same time, there are other interpretations of the DISC results. While the projection from the hippocampus to the prefrontal cortex is not directly reciprocated, there is a potential pathway from the prefrontal cortex to the hippocampus that involves a relay in the nucleus reuniens (Wouterlood et al., 1990; McKenna and Vertes, 2004; Vertes et al., 2006; Prasad et al., 2011). We have recently shown that damage to this midline thalamic structure gives rise to inhibitory control deficits, especially in impulse control (Prasad et al., 2012). Thus, it is possible that indirect prefrontal input to the hippocampus through the thalamus is the disruption that causes premature responding, and perhaps perseveration, in the DISC group of rats. Furthermore, while lesions of the orbital and prelimbic cortex increase perseveration, only lesions of the infralimbic cortex increase premature responding. It is feasible, therefore, that the input to the nucleus reuniens from different prefrontal areas may indirectly support the control of behavior expressed by the $\mathrm{vHC}$.

Finally, it is possible that the interaction between vHC and vPFC stems not from direct or indirect projections between the two areas, but instead from the control that the two areas exert over the mesolimbic pathway via projection, for example, to the ventral striatum. Both the vPFC and ventral CA1/subiculum project to the nucleus accumbens (Groenewegen et al., 1987; Brog et al., 1993) where they converge with dopaminergic neurons arising from the ventral tegmental area (Yang and Mogenson, 1984; Totterdell and Smith, 1989; Sesack and Pickel, 1990). Thus, both projections are thought to be important determinants of nucleus accumbens activity, and therein regulate the firing of ventral tegmental area dopamine neurons (Floresco et al., 2001; Grace et al., 2007; Belujon and Grace, 2008). Given that bilateral lesions of the nucleus accumbens (Christakou et al., 2004) and prefrontalaccumbens disconnections (Floresco et al., 1999; Christakou et al., 2004; Block et al., 2007) both lead to increases in perseverative responding; the hippocampal-prefrontal disconnection results presented here might suggest that the observed perseverative deficits more closely reflect disruption in the corticostriatal projec- 
tions rather than the corticocortical or corticothalamic projections. Whether dopamine dysregulation per se is involved in inhibitory control deficits is presently unclear. However, since hippocampal lesions are known to increase dopamine release in the nucleus accumbens (Campbell et al., 1971; Lipska et al., 1991, 1992; Wilkinson et al., 1993), and intra-accumbens stimulation of dopamine receptors increases both premature and perseverative responses (Pezze et al., 2007), this interpretation is certainly plausible (for review, see Goto and Grace, 2008). The finding that systemic dopamine stimulation exaggerates the perseverative effects of bilateral vHC lesions is also consistent with this hypothesis (Abela et al., 2012).

\section{Concluding remarks}

In summary, our data provide evidence for the interdependent function and conjoint importance of the $\mathrm{vHC}$ and $\mathrm{vPFC}$ in response inhibition. By implication, the normal interaction between these structures may function to suppress the execution of inappropriate actions to optimize complex executive behavior. Such deficits of executive control are symptoms of neuropsychiatric illness including schizophrenia, obsessivecompulsive disorder, and pervasive developmental disorders such as attention-deficit hyperactivity disorder (Solanto, 1998; Pantelis et al., 2004; Menzies et al., 2008). Our data reinforce the possibility that hippocampal and prefrontal cortical circuits are being compromised in these different disorders, at least with respect to response control.

\section{References}

Abela AR, Dougherty SD, Fagen ED, Hill C, Chudasama Y (2012) Inhibitory control deficits in rats with ventral hippocampal lesions. Cereb Cortex, in press.

Belujon P, Grace AA (2008) Critical role of the prefrontal cortex in the regulation of hippocampus-accumbens information flow. J Neurosci 28:9797-9805.

Block AE, Dhanji H, Thompson-Tardif SF, Floresco SB (2007) Thalamicprefrontal cortical-ventral striatal circuitry mediates dissociable components of strategy set shifting. Cereb Cortex 17:1625-1636.

Brog JS, Salyapongse A, Deutch AY, Zahm DS (1993) The patterns of afferent innervation of the core and shell in the "accumbens" part of the rat ventral striatum: immunohistochemical detection of retrogradely transported fluoro-gold. J Comp Neurol 338:255-278.

Campbell BA, Ballantine P 2nd, Lynch G (1971) Hippocampal control of behavioral arousal: duration of lesion effects and possible interactions with recovery after frontal cortical damage. Exp Neurol 33:159-170.

Cardinal RN, Aitken MR (2010) Whisker: a client-server high-performance multimedia research control system. Behav Res Methods 42:1059-1071.

Christakou A, Robbins TW, Everitt BJ (2004) Prefrontal cortical-ventral striatal interactions involved in affective modulation of attentional performance: implications for corticostriatal circuit function. J Neurosci 24:773-780.

Chudasama Y (2011) Animal models of prefrontal-executive function. Behav Neurosci 125:327-343.

Chudasama Y, Muir JL (2001) Visual attention in the rat: a role for the prelimbic cortex and thalamic nuclei? Behav Neurosci 115:417-428.

Chudasama Y, Passetti F, Rhodes SE, Lopian D, Desai A, Robbins TW (2003) Dissociable aspects of performance on the 5-choice serial reaction time task following lesions of the dorsal anterior cingulate, infralimbic and orbitofrontal cortex in the rat: differential effects on selectivity, impulsivity and compulsivity. Behav Brain Res 146:105-119.

Dalley JW, Everitt BJ, Robbins TW (2011) Impulsivity, compulsivity, and top-down cognitive control. Neuron 69:680-694.

Dunnett SB, Meldrum A, Muir JL (2005) Frontal-striatal disconnection disrupts cognitive performance of the frontal-type in the rat. Neuroscience 135:1055-1065.

Floresco SB, Braaksma DN, Phillips AG (1999) Thalamic-cortical-striatal circuitry subserves working memory during delayed responding on a radial arm maze. J Neurosci 19:11061-11071.
Floresco SB, Todd CL, Grace AA (2001) Glutamatergic afferents from the hippocampus to the nucleus accumbens regulate activity of ventral tegmental area dopamine neurons. J Neurosci 21:4915-4922.

French SJ, Totterdell S (2002) Hippocampal and prefrontal cortical inputs monosynaptically converge with individual projection neurons of the nucleus accumbens. J Comp Neurol 446:151-165.

French SJ, Totterdell S (2003) Individual nucleus accumbens-projection neurons receive both basolateral amygdala and ventral subicular afferents in rats. Neuroscience 119:19-31.

Goto Y, Grace AA (2008) Limbic and cortical information processing in the nucleus accumbens. Trends Neurosci 31:552-558.

Grace AA, Floresco SB, Goto Y, Lodge DJ (2007) Regulation of firing of dopaminergic neurons and control of goal-directed behaviors. Trends Neurosci 30:220-227.

Gray JA, McNaughton N (1983) Comparison between the behavioural effects of septal and hippocampal lesions: a review. Neurosci Biobehav Rev $7: 119-188$.

Groenewegen HJ, Vermeulen-Van der Zee E, te Kortschot A, Witter MP (1987) Organization of the projections from the subiculum to the ventral striatum in the rat. A study using anterograde transport of Phaseolus vulgaris leucoagglutinin. Neuroscience 23:103-120.

Heidbreder CA, Groenewegen HJ (2003) The medial prefrontal cortex in the rat: evidence for a dorso-ventral distinction based upon functional and anatomical characteristics. Neurosci Biobehav Rev 27:555-579.

Ishikawa A, Nakamura S (2006) Ventral hippocampal neurons project axons simultaneously to the medial prefrontal cortex and amygdala in the rat. J Neurophysiol 96:2134-2138.

Jarrard LE, Isaacson RL (1965) Hippocampal ablation in rats: effects of intertrial interval. Nature 207:109-110.

Jay TM, Witter MP (1991) Distribution of hippocampal CA1 and subicular efferents in the prefrontal cortex of the rat studied by means of anterograde transport of Phaseolus vulgaris-leucoagglutinin. J Comp Neurol 313:574-586.

Lipska BK, Jaskiw GE, Karoum F, Phillips I, Kleinman JE, Weinberger DR (1991) Dorsal hippocampal lesion does not affect dopaminergic indices in the basal ganglia. Pharmacol Biochem Behav 40:181-184.

Lipska BK, Jaskiw GE, Chrapusta S, Karoum F, Weinberger DR (1992) Ibotenic acid lesion of the ventral hippocampus differentially affects dopamine and its metabolites in the nucleus accumbens and prefrontal cortex in the rat. Brain Res 585:1-6.

McKenna JT, Vertes RP (2004) Afferent projections to nucleus reuniens of the thalamus. J Comp Neurol 480:115-142.

Menzies L, Chamberlain SR, Laird AR, Thelen SM, Sahakian BJ, Bullmore ET (2008) Integrating evidence from neuroimaging and neuropsychological studies of obsessive-compulsive disorder: the orbitofronto-striatal model revisited. Neurosci Biobehav Rev 32:525-549.

Murphy ER, Dalley JW, Robbins TW (2005) Local glutamate receptor antagonism in the rat prefrontal cortex disrupts response inhibition in a visuospatial attentional task. Psychopharmacology (Berl) 179:99-107.

Nonneman AJ, Voigt J, Kolb BE (1974) Comparisons of behavioral effects of hippocampal and prefrontal cortex lesions in the rat. J Comp Physiol Psychol 87:249-260.

Pantelis C, Harvey CA, Plant G, Fossey E, Maruff P, Stuart GW, Brewer WJ, Nelson HE, Robbins TW, Barnes TR (2004) Relationship of behavioural and symptomatic syndromes in schizophrenia to spatial working memory and attentional set-shifting ability. Psychol Med 34:693-703.

Passetti F, Chudasama Y, Robbins TW (2002) The frontal cortex of the rat and visual attentional performance: dissociable functions of distinct medial prefrontal subregions. Cereb Cortex 12:1254-1268.

Paxinos G, Watson C (2005) The rat brain in stereotaxic coordinates, Ed 5. Burlington, MA: Elsevier Academic.

Pezze MA, Dalley JW, Robbins TW (2007) Differential roles of dopamine D1 and D2 receptors in the nucleus accumbens in attentional performance on the five-choice serial reaction time task. Neuropsychopharmacology 32:273-283.

Prasad JA, Antkowiak P, Chudasama Y (2011) Interconnected pathway between rat ventral prefrontal cortex, thalamic reuniens and ventral hippocampus revealed by retrograde transport of pseudorabies virus. Soc Neurosci Abstr 37:511.09.

Prasad JA, Macgregor EM, Chudasama Y (2012) Lesions of the thalamic reuniens cause impulsive but not compulsive responses. Brain Struct Funct, in press. Rawlins JN, Feldon J, Ursin H, Gray JA (1985) Resistance to extinction after 
schedules of partial delay or partial reinforcement in rats with hippocampal lesions. Exp Brain Res 59:273-281.

Sesack SR, Pickel VM (1990) In the rat medial nucleus accumbens, hippocampal and catecholaminergic terminals converge on spiny neurons and are in apposition to each other. Brain Res 527:266-279.

Solanto MV (1998) Neuropsychopharmacological mechanisms of stimulant drug action in attention-deficit hyperactivity disorder: a review and integration. Behav Brain Res 94:127-152.

Thierry AM, Gioanni Y, Dégénétais E, Glowinski J (2000) Hippocampoprefrontal cortex pathway: anatomical and electrophysiological characteristics. Hippocampus 10:411-419.

Totterdell S, Smith AD (1989) Convergence of hippocampal and dopaminergic input onto identified neurons in the nucleus accumbens of the rat. J Chem Neuroanat 2:285-298.

Vertes RP, Hoover WB, Do Valle AC, Sherman A, Rodriguez JJ (2006) Efferent projections of reuniens and rhomboid nuclei of the thalamus in the rat. J Comp Neurol 499:768-796.
Verwer RW, Meijer RJ, Van Uum HF, Witter MP (1997) Collateral projections from the rat hippocampal formation to the lateral and medial prefrontal cortex. Hippocampus 7:397-402.

Wilkinson LS, Mittleman G, Torres E, Humby T, Hall FS, Robbins TW (1993) Enhancement of amphetamine-induced locomotor activity and dopamine release in nucleus accumbens following excitotoxic lesions of the hippocampus. Behav Brain Res 55:143-150.

Winer BJ (1971) Statistical principles in experimental design, Ed 2. New York: McGraw-Hill.

Wouterlood FG, Saldana E, Witter MP (1990) Projection from the nucleus reuniens thalami to the hippocampal region: light and electron microscopic tracing study in the rat with the anterograde tracer Phaseolus vulgaris-leucoagglutinin. J Comp Neurol 296:179-203.

Yang CR, Mogenson GJ (1984) Electrophysiological responses of neurones in the nucleus accumbens to hippocampal stimulation and the attenuation of the excitatory responses by the mesolimbic dopaminergic system. Brain Res 324:69-84. 